\title{
A nationwide analysis on the influence of obesity in inflammatory bowel disease hospitalizations
}

\author{
Dushyant Singh Dahiya ${ }^{1}$, Asim Kichloo ${ }^{1,2}$, Farah Wani ${ }^{3}$, Jagmeet Singh ${ }^{4}$, Dhanshree Solanki ${ }^{5}$, Hafeez Shaka ${ }^{6}$ \\ ${ }^{I}$ Department of Internal Medicine, Central Michigan University College of Medicine, Saginaw, MI; Departments of ${ }^{2}$ Internal Medicine and \\ ${ }^{3}$ Family Medicine, Samaritan Medical Center, Watertown, NY; ${ }^{4}$ Department of Internal Medicine, Geisinger School of Medicine, Scranton, PA; \\ ${ }^{5}$ Department of Internal Medicine, Rutgers University, New Brunswick, NJ; ${ }^{6}$ Department of Internal Medicine, John H. Stroger, Jr. Hospital of \\ Cook County, Chicago, IL, USA
}

Background/Aims: Proinflammatory cytokines released from adipocytes can influence the development, progression, and treatment of inflammatory bowel disease (IBD), and may be associated with worse clinical outcomes. Methods: For 20162018, we analyzed data from the Nationwide Inpatient Sample to identify adult ( $\geq 18$ years) hospitalizations with a primary discharge diagnosis of IBD. The study sample was divided based on the presence or absence of obesity. The primary outcomes included inpatient mortality, while the secondary outcomes consisted of system-based complications and disease implications on the United States healthcare system. Results: We identified 282,005 hospitalizations of IBD from 2016 to 2018. Of these hospitalizations, 26,465 (9.4\%) had a secondary diagnosis of obesity while 255,540 (90.6\%) served as controls. IBD hospitalizations with obesity had a higher mean age ( 47.9 years vs. 45.2 years, $P<0.001$ ), middle age (range, $40-65$ years) predominance (37.7\% vs. $28.9 \%, P<0.001)$, female predominance $(64.1 \%$ vs. $52.5 \%, P<0.001)$ and higher proportion of patients with comorbidities compared to the non-obese cohort. White predominance was observed in both subgroups. No difference in the odds of inpatient mortality was noted between the 2 subgroups; however, IBD hospitalizations with obesity had higher mean total hospital charge ( $\$ 50,126$ vs. $\$ 45,001, P<0.001$ ), longer length of stay (5.5 days vs. 4.9 days, $P<0.001$ ) and higher proportion of complications compared to the non-obese cohort. Conclusions: Obese IBD hospitalizations had higher length of stay, total hospital charge, and complications compared to the non-obese cohort. (Intest Res 2022;20:342-349)

Key Words: Inflammatory bowel disease; Obesity; Epidemiology; Outcomes; Nationwide Inpatient Sample

\section{INTRODUCTION}

Inflammatory bowel disease (IBD) is a broad term characterized by the presence of chronic inflammation in the gastrointestinal tract which results from host-microbial interactions in individuals who are genetically susceptible. ${ }^{1}$ It is an autoimmune condition which can be further subdivided into 2 distinct entities, namely ulcerative colitis (UC) and Crohn's dis-

Received March 17, 2021. Revised May 4, 2021. Accepted May 5, 2021. Correspondence to Dushyant Singh Dahiya, Department of Internal Medicine, Central Michigan University College of Medicine, 1000 Houghton Avenue, Saginaw, MI 48602, USA. Tel: +1-678-602-1176, Fax: +1-315-7854089, E-mail: dush.dahiya@gmail.com ease (CD), based on the pattern of mucosal involvement and characteristics of the chronic inflammatory process. ${ }^{2}$ The exact etiology of IBD is currently unknown; however, it is hypothesized that immune response dysregulation, abnormal gut microbiota, environmental factors, and genetics may play a key role in pathogenesis. ${ }^{3}$ IBD may not be a leading cause of mortality in the United States (US) or worldwide, but its rising prevalence places substantial burden on the healthcare systems while also negatively impacting the patient's quality of life. ${ }^{4,5}$ Obesity, defined as abnormal or excessive fat accumulation for a given height (body mass index $[\mathrm{BMI}] \geq 30 \mathrm{~kg} / \mathrm{m}^{2}$ ), has reached epidemic status in the US and IBD is being increasingly diagnosed in these individuals. ${ }^{6}$ It has been recognized 
as a state of chronic low-grade inflammation with constant release of proinflammatory cytokines from the adipocytes. ${ }^{7} \mathrm{Al}-$ though researchers are actively investigating the exact mechanism implicated in the development of IBD in obese patients, there is significant paucity of data on the impact of obesity in IBD patients admitted to the hospital. The main objective of our study was to help bridge this gap in knowledge. In our study, we used the National Inpatient Sample (NIS) database to determine the epidemiology, demographic distribution, adverse outcomes, and system-based complications of IBD hospitalizations with obesity. We also further compare these characteristics with non-obese IBD hospitalizations which served as a control population. This comparative study designed helped us estimate the influence of obesity on IBD hospitalizations. Furthermore, we also discuss the geographical distribution of the disease entity across the US and detail the burden of the disease on the US healthcare system in terms of healthcare cost and resource utilization for the study period.

\section{METHODS}

\section{Design and Data Source}

This was a retrospective cohort study design involving all adult hospitalizations with IBD in the US from 2016 to 2018. Data was extracted from the NIS database using the Stata ${ }^{\circledR}$ version 16 software (StataCorp, College Station, TX, USA) for the study period. The NIS database was developed by the Healthcare Cost and Utilization Project (HCUP), a Federal-State-Industry partnership sponsored by the Agency for Healthcare Research and Quality. It is derived from billing data submitted by individual hospitals across the US to statewide data organizations, covering 97\% of the US population. ${ }^{8}$ Excluding rehabilitation centers and long-term acute care hospitals, it approximates a $20 \%$ stratified sample of discharges from US community hospitals. The dataset is further weighted to obtain national estimates. ${ }^{9}$ The 2016 to 2018 databases were coded using the International Classification of Diseases, 10th Revision, Clinical Modification/Procedure Coding System (ICD-10-CM/PCS). In NIS, codes are divided into a principal diagnosis which is the main ICD-10 code and secondary diagnoses which consist of any ICD-10 code other than the code for the principal diagnosis.

\section{Study Population}

The study population included all adult ( $\geq 18$ years) hospitalizations with a primary discharge diagnosis of IBD, including both UC and CD, from 2016 to 2018. These were classified as "IBD hospitalizations" for the study. Individuals less than 18 years of age were excluded from the study. This cohort was further subdivided into 2 groups based on the presence or absence of a secondary diagnosis of obesity, defined as a BMI $>30 \mathrm{~kg} / \mathrm{m}^{2}$, using the ICD codes available from the Clinical Classifications Software Refined, developed and standardized by HCUP, were used. ${ }^{10}$

\section{Outcome Measures}

The primary outcome was comparing inpatient mortality between patients with IBD and obesity, to those without obesity. Secondary outcomes included comparing rates of acute myocardial infarction, sepsis, need for transfusion, acute renal failure, acute respiratory failure (ARF), pulmonary embolism (PE) as well as mean length of hospitalization (LOS), and mean total hospital charges (THC).

\section{Statistical Analysis}

A thorough analysis was performed using the Stata ${ }^{\circledR}$ version 16 software (StataCorp). All analyses were conducted using weighted samples for national estimates and were in adjunct with HCUP regulations. Comorbidities were calculated as proportions and the chi-square test was used to compare these characteristics between the 2 groups. A multivariate regression analysis was done to adjust for possible confounders while calculating the primary and secondary outcomes. The confounders were obtained after a thorough literature review and consisted of age, sex, race, hospital bed size, location and teaching status and grouped Charlson Comorbidity Index. A negative binomial regression model was used to adjust for count data including LOS and THC, expressed as incidence rate ratio (IRR). A threshold of 0.05 was maintained for statistical significance of the outcomes.

\section{Ethical Considerations}

The NIS database lacks patient specific and hospital level identifiers. Our study was conducted with Institutional Review Board approval from Central Michigan University College of Medicine. As the NIS database lacks patient identifiers, informed consent was waived.

\section{Data Availability Statement}

The data for the study was obtained from the NIS which is a large publicly available all-payer inpatient care database containing information on more than 7 million hospital stays across 
Table 1. Characteristics of IBD Hospitalizations with and without the Presence of Obesity

\begin{tabular}{|c|c|c|c|}
\hline Variable & $\begin{array}{l}\text { IBD with } \\
\text { obesity (\%) }\end{array}$ & $\begin{array}{l}\text { IBD without } \\
\text { obesity (\%) }\end{array}$ & $P$-value \\
\hline $\begin{array}{l}\text { Sample size }(n=282,005) \text {, } \\
\text { No. }(\%)\end{array}$ & $26,465(9.4)$ & $255,540(90.6)$ & \\
\hline Crohn's disease & 59.7 & 62.5 & $<0.001$ \\
\hline Ulcerative colitis & 40.3 & 37.5 & $<0.001$ \\
\hline Women sex & 64.1 & 52.5 & $<0.001$ \\
\hline Mean age (yr) & 47.9 & 45.2 & $<0.001$ \\
\hline Age categories (yr) & & & $<0.001$ \\
\hline $18-39$ & 45.2 & 53.6 & \\
\hline $40-64$ & 37.7 & 28.9 & \\
\hline$\geq 65$ & 17.1 & 17.5 & \\
\hline Racial distribution & & & 0.009 \\
\hline Caucasian & 70.5 & 70.6 & \\
\hline African American & 15.0 & 13.5 & \\
\hline Hispanic & 7.7 & 7.9 & \\
\hline Others & 6.8 & 8.0 & \\
\hline CCl score & & & $<0.001$ \\
\hline 0 & 16.2 & 23.8 & \\
\hline 1 & 8.5 & 6.1 & \\
\hline 2 & 3.7 & 2.3 & \\
\hline$\geq 3$ & 71.6 & 67.8 & \\
\hline Insurance type & & & $<0.001$ \\
\hline Medicaid & 29.7 & 26.3 & \\
\hline Medicare & 19.7 & 19.6 & \\
\hline Private & 46.1 & 48.3 & \\
\hline Uninsured & 4.5 & 5.8 & \\
\hline \multicolumn{3}{|c|}{ Median annual income in patient's zip code (USD) } & $<0.001$ \\
\hline $1-43,999$ & 27.9 & 25.5 & \\
\hline $44,000-55,999$ & 27.1 & 25.3 & \\
\hline $56,000-73,999$ & 27.2 & 25.3 & \\
\hline$\geq 74,000$ & 17.8 & 23.9 & \\
\hline \multicolumn{4}{|l|}{ Comorbidities } \\
\hline Diabetes mellitus & 21.7 & 8.2 & $<0.001$ \\
\hline Hypertension & 39.0 & 22.5 & $<0.001$ \\
\hline Smoking history & 40.6 & 36.8 & $<0.001$ \\
\hline Congestive heart failure & 5.6 & 2.5 & $<0.001$ \\
\hline Chronic kidney disease & 7.4 & 4.4 & $<0.001$ \\
\hline Dyslipidemia & 23.3 & 12.7 & $<0.001$ \\
\hline Coronary artery disease & 8.2 & 5.8 & $<0.001$ \\
\hline $\begin{array}{l}\text { Chronic obstructive } \\
\text { pulmonary disease }\end{array}$ & 7.8 & 5.2 & $<0.001$ \\
\hline
\end{tabular}

(Continued to the next)
Table 1. Continued

\begin{tabular}{|c|c|c|c|}
\hline Variable & $\begin{array}{l}\text { IBD with } \\
\text { obesity (\%) }\end{array}$ & $\begin{array}{l}\text { IBD without } \\
\text { obesity (\%) }\end{array}$ & $P$-value \\
\hline Malnutrition & 10.6 & 13.6 & $<0.001$ \\
\hline History of neoplasm & 6.8 & 6.8 & 0.855 \\
\hline History of anemia & 37.2 & 37.8 & 0.419 \\
\hline \multicolumn{4}{|l|}{ Hospital characteristics } \\
\hline Hospital region & & & $<0.001$ \\
\hline Northeast & 19.4 & 21.7 & \\
\hline Midwest & 28.8 & 23.9 & \\
\hline South & 37.9 & 37.9 & \\
\hline West & 13.9 & 16.5 & \\
\hline Hospital bed size & & & 0.190 \\
\hline Small & 19.8 & 18.8 & \\
\hline Medium & 28.5 & 28.1 & \\
\hline Large & 51.7 & 53.1 & \\
\hline Urban location & 93.6 & 93.1 & 0.156 \\
\hline Teaching hospital & 74.1 & 72.2 & 0.010 \\
\hline
\end{tabular}

IBD, inflammatory bowel disease; $\mathrm{CCl}$, Charlson Comorbidity Index.

the US. The large sample size available from the NIS is ideal for developing national and regional estimates and enables analysis of large sets of population.

\section{RESULTS}

\section{Biodemographic Characteristics}

From 2016 to 2018, we identified 282,005 hospitalizations of IBD, including both UC and CD. Of these hospitalizations, 26,465 patients $(9.4 \%)$ had a secondary diagnosis of obesity while 255,540 patients (90.6\%) served as controls. IBD hospitalizations with obesity were noted to be significantly older (47.9 years vs. 45.2 years, $P<0.001$ ) with a middle age (range, $40-65$ years) ( $37.7 \%$ vs. $28.9 \%, P<0.001)$ and female predominance $(64.1 \%$ vs. $52.5 \%, P<0.001)$ compared to patients in the non-obese subgroup. For IBD hospitalizations with obesity, Caucasians made up $70.5 \%$ of the study population followed by African American (15\%), Hispanics (7.7\%) and other races (6.8\%). Similarly, for IBD hospitalizations without obesity, a Caucasian predominance $(70.6 \%)$ in the study population was noted followed by African American (13.5\%), Hispanics (7.9\%) and other races (8\%). Compared to IBD hospitalizations without obesity, we noted a higher percentage of patients with comorbidities such as diabetes mellitus $(21.7 \%$ vs. $8.2 \%, P<0.001)$, dyslipidemia ( $23.3 \%$ vs. $12.7 \%, P<0.001)$, cor- 
Table 2. Clinical Outcomes for IBD Hospitalizations with and without Obesity

\begin{tabular}{lcccc}
\hline Outcome & $\begin{array}{c}\text { IBD with obesity } \\
(\%)\end{array}$ & $\begin{array}{c}\text { IBD without obesity } \\
(\%)\end{array}$ & aOR (95\% CI) & $P_{\text {-value }}$ \\
\hline $\begin{array}{l}\text { Sample size (n=282,005), No. (\%) } \\
\text { Primary outcome }\end{array}$ & $26,465(9.4)$ & $255,540(90.6)$ & & \\
$\quad$ Inpatient mortality & 0.28 & 0.30 & $1.03(0.60-1.77)$ & 0.904 \\
Secondary outcomes & & & & \\
$\quad$ Mean length of stay & 5.5 & 4.9 & $1.12^{\mathrm{b}}(1.09-1.16)$ & $<0.001^{\mathrm{a}}$ \\
$\quad$ Mean total hospital charge (USD) & 50,126 & 45,001 & $1.32^{\mathrm{b}}(1.20-1.46)$ & $<0.001^{\mathrm{a}}$ \\
Sepsis & 1.5 & 1.2 & $1.34(1.05-1.71)$ & $0.018^{\mathrm{a}}$ \\
Acute myocardial infarction & 0.2 & 0.2 & $1.15(0.61-2.66)$ & 0.666 \\
$\quad$ Transfusion of blood products & 5.3 & 5.8 & $0.92(0.81-1.05)$ & 0.201 \\
$\quad$ Acute kidney failure & 10.2 & 7.5 & $1.37(1.24-1.51)$ & $<0.001^{\mathrm{a}}$ \\
Acute respiratory failure & 0.9 & 0.6 & $1.43(1.05-1.96)$ & $0.025^{\mathrm{a}}$ \\
Acute pulmonary embolism & 0.6 & 0.4 & $1.47(1.01-2.17)$ & $0.049^{\mathrm{a}}$ \\
\hline
\end{tabular}

${ }^{a}$ Statistically significant, $P<0.05$.

${ }^{b}$ Adjusted incidence rate ratio.

$\mathrm{IBD}$, inflammatory bowel disease; $\mathrm{aOR}$, adjusted odds ratio; $\mathrm{Cl}$, confidence interval.

onary artery disease ( $8.2 \%$ vs. $5.8 \%, P<0.001)$, hypertension (39\% vs. $22.5 \%, P<0.001$ ), congestive heart failure (5.6\% vs. $2.5 \%$, $P<0.001)$, chronic kidney disease ( $7.4 \%$ vs. $4.4 \%, P<0.001)$, chronic obstructive pulmonary disease ( $7.8 \%$ vs. $5.2 \%, P<0.001$ ), and history of smoking ( $40.6 \%$ vs. $36.8 \%, P<0.001$ ) in IBD hospitalizations with obesity. However, IBD hospitalizations without obesity had more patients with malnutrition (13.6\% vs. $10.6 \%, P<0.001$ ) than the obesity subgroup (Table 1 ).

\section{Hospital Characteristics}

A higher proportion of IBD hospitalizations with obesity were noted in the South (37.9\%) hospital region, followed by the Midwest (28.8\%), Northeast (19.4\%) and West (13.9\%). Most of these hospitalizations were in teaching hospitals (74.1\%). Similarly, for IBD hospitalizations without obesity, the highest proportion of hospitalizations were seen in the South (37.9\%), followed by the Midwest (23.9\%), Northeast (21.7\%) and West (16.5\%) hospital regions (Table 1).

\section{Primary Outcome}

We did not find any statistical significance in the odds of inpatient mortality ( $0.28 \%$ vs. 0.30\%: adjusted odds ratio [aOR], 1.03; 95\% confidence interval [CI], 0.60-1.77; $P=0.904$ ) between IBD hospitalizations with and without obesity (Table 2).

\section{Secondary Outcomes}

IBD hospitalizations with obesity were noted to have longer LOS (5.5 days vs. 4.9 days: IRR, 1.12; 95\% CI, 1.09-1.16; $P<0.001$ ) and higher mean THC ( $\$ 50,126$ vs. $\$ 45,001$ : IRR, 1.32; 95\% CI, 1.20-1.46; $P<0.001)$ compared to patients without obesity (Table 2). Furthermore, inpatient complications such as sepsis (1.5\% vs. 1.2\%: aOR, 1.34; 95\% CI, 1.05-1.71; $P=0.018$ ), acute kidney failure (AKF) (10.2\% vs. 7.5\%: aOR, 1.37; 95\% CI, 1.24$1.51 ; P<0.001)$, ARF (0.9\% vs. $0.6 \%$ : aOR, $1.43 ; 95 \%$ CI, $1.05-$ $1.96 ; P=0.025)$, and PE ( $0.6 \%$ vs. $0.4 \%$ : aOR, $1.47 ; 95 \% \mathrm{CI}, 1.01-$ 2.17; $P=0.049)$ were more frequent for IBD hospitalizations with obesity compared to those without (Table 2). From a payment perspective for IBD hospitalizations with obesity, private insurance was the largest payer (46.1\%) followed by Medicaid (29.7\%) and Medicare (19.7\%). About 4.5\% of these patients were uninsured. Additionally, a majority of IBD hospitalizations with obesity were in the $\$ 1-\$ 43,999$ (low) median annual income group (27.7\%), followed by the $\$ 56,000-\$ 73,999$ (27.2\%), $\$ 44,000-\$ 55,999(27.1 \%)$ and $\geq \$ 74,000$ (17.8\%) median annual income groups $(P<0.001)$.

\section{DISCUSSION}

IBD is a broad term used to describe states of chronic progressive inflammation of the mucosa of the gastrointestinal tract in individuals who are genetically susceptible. ${ }^{11}$ The prevalence 
of IBD continues to be on the rise around the world with literature reporting an increase in the cases of IBD by $85.1 \%$ from 1990 to 2017 . $^{4}$ Similar trends have been observed in the US with the Centers for Disease Control and Prevention (CDC) reporting an increase in the prevalence of IBD from 2 million $(0.9 \%)$ in 1999 to 3 million (1.3\%) cases in $2015 .{ }^{4}$ Although IBD may not a leading cause of mortality, its rising prevalence, both around the world and in the US, is concerning as it significantly impacts the patient's quality of life. ${ }^{12}$ Furthermore, over the last few decades, obesity has emerged as a global pandemic with rising prevalence. ${ }^{13}$ The US has seen a doubling of the rates of obesity since the 1970s with over two-thirds of the American population being classified as overweight and one-third as obese. ${ }^{14}$ The IBD population, which has historically been associated with malnourishment, is not immune to the rising trend of obesity with studies reporting increased diagnosis of IBD in obese populations. ${ }^{6}$ The presence of obesity in IBD is an area of particular interest as it may lead to worse clinical outcomes and place undue burden on the healthcare system across the globe. Although current literature describes the association between obesity and IBD, there is significant gap in knowledge on numerous aspects of the disease entity in an inpatient setting. Therefore, this study was designed to investigate and compare the biodemographic characteristics, associations, inpatient mortality, and numerous system-based complications of obesity in IBD hospitalizations. Furthermore, we also determined the distribution of the disease entity and its burden on the US healthcare system in terms of costs and healthcare utilization.

Numerous cross-sectional studies have demonstrated that about $20 \%-40 \%$ of patients with IBD are overweight and around $15 \%-40 \%$ are obese. ${ }^{15}$ In our study, we identified 282,005 hospitalizations of IBD using NIS for the 2016 to 2018 period. We report that $9.4 \%$ of these hospitalizations had a secondary diagnosis of obesity. This prevalence rate was lower than that reported in literature. It may be explained by the fact that due to increasing awareness about the disease entity, there is early recognition and intervention by physician providers, researchers, community organizations and individuals themselves. ${ }^{16}$ IBD hospitalizations with obesity were significantly older with a mean age of 47.9 years compared to those without obesity. This may be because an increasing age is associated with insulin resistance and dysregulated adipokine secretion which may increase fat mass accumulation and redistribution. ${ }^{17}$ This may lead to an increase in the incidence of obesity with increasing age which promotes the development of IBD. Addition- ally, a large-scale detection power of the study may also, in part, have had a role to play in the differences noted for mean age. Furthermore, literature has described gender disparities both in the prevalence of IBD and obesity. ${ }^{18,19}$ As per the CDC, the age-adjusted prevalence of severe obesity was higher in women compared to men. ${ }^{18}$ Our study reflects these findings as we note a significant female predominance in IBD hospitalizations with obesity. Additionally, racial differences were noted between obese and non-obese IBD hospitalizations. However, Caucasians made up most of the study population in both cohorts. This may because IBD is more commonly seen in Caucasians compared to any other race. ${ }^{20}$

Obesity is associated with significant morbidity and mortality particularly from cardiovascular causes and cancers. ${ }^{21}$ The relationship between obesity and mortality varies substantially with the patient population, BMI, and the cause of death, and may change over time. ${ }^{21,22}$ Literature reports that the obesity-related age-adjusted mortality rates have increased by 142\% from 1999 to 2016 in the US disproportionately affecting men compared to women. ${ }^{23}$ For patients with IBD, there is significant paucity of data on the exact mortality rates as the death may be secondary to a complication of IBD rather than the disease itself. As per literature, the 30-day mortality rate for CD and UC have been estimated to be 2.7 and 3.8 per 100 hospital stays, respectively. ${ }^{24}$ Based on poor clinical outcomes associated with obesity in other chronic inflammatory diseases, it is extrapolated that obesity potentially leads to worse clinical outcomes in patients with IBD, but the studies exploring this association in-depth provide conflicting evidence. ${ }^{25,26}$ In our study, the mortality rate for IBD hospitalizations with obesity was found to be $0.28 \%$. However, we did not find any statistical significance in the odds of inpatient mortality between obese and non-obese IBD hospitalizations. The exact reason for this finding is unknown but as discussed earlier it may be because these patients die due to causes other than IBD. Furthermore, we also assessed system-based inpatient complications of these IBD hospitalizations. Compared to the non-obese cohort, IBD hospitalizations with obesity had higher proportion of patients with complications such as sepsis, AKF, ARF, and PE. Nonetheless, we advocate for the need of additional large, prospective, multi-center studies to investigate the outcomes associated with IBD hospitalizations in the presence of obesity and to further evaluate the benefit of weight reduction in these patients.

In recent years, numerous studies have estimated the burden of IBD on the US healthcare system in terms of costs and 
healthcare utilization. In 2014, the mean charge for inpatient management of CD was noted to be $\$ 11,345$ and $\$ 13,412$ for UC. ${ }^{27}$ From 2006 to 2015, the mean LOS for IBD hospitalizations was noted to be 6.7 days with a standard deviation of 5.8 days. ${ }^{28}$ In our study, IBD hospitalizations with obesity were noted to have a mean THC of $\$ 50,126$ and LOS of 5.5 days as compared to the $\$ 45,001$ and 4.9 days for the non-obese cohort, respectively. Therefore, the presence of obesity is associated with higher costs and longer length of hospital stay in IBD hospitalizations. Hence, in these patients it becomes essential to educate and promote dietary and lifestyle changes to potentially decrease inpatient admissions and the burden of the disease on the healthcare system. Frequent outpatient followup with goal-oriented weight reduction may also be beneficial in these patients. Furthermore, we also assessed the geographical distribution of these hospitalizations across the US using the NIS database. ${ }^{29,30}$ We noted higher proportion of IBD hospitalizations with obesity in the South, followed by the Midwest, Northeast and West hospital regions. This distribution may be because the Southern hospital regions have a higher prevalence of obesity and IBD compared to other regions of the US. ${ }^{31}$ For IBD hospitalizations with obesity, from a payment perspective, private insurance was the largest payer followed by Medicaid and Medicare. Only $4.5 \%$ of these patients were uninsured. Additionally, a majority of IBD hospitalizations with obesity were in the low median annual income $(\$ 1-\$ 43,999)$ subgroup. This may be due to consumption of low-quality, highfat foods due to economic restraints. ${ }^{32}$

This study has several strengths along with limitations. The biggest strength of our study is the study population, which is derived from a large, national, publicly available database developed through a Federal-State-Industry partnership and contains data on inpatient admissions from all over the US. Hence, the outcomes of the study are applicable to all hospitalizations of IBD across the US. Furthermore, the study design helps us investigate, in-depth, the epidemiology, adverse outcomes, complications, and the consequences on the healthcare system for IBD hospitalizations with obesity while also comparing it with a control population. This allows for a comprehensive analysis which adds meaningful data to current literature.

However, we do acknowledge the limitations associated with our study. The NIS database lacks data on the severity of the disease and time from the diagnosis of obesity to the development of IBD. Furthermore, it does not contain data on medications used by patients with IBD or their dosage. We also acknowledge all the all the limitations associated with using ICD codes for obesity, which was defined as a BMI $>30$ $\mathrm{kg} / \mathrm{m}^{2}$ in adults per the NIS database. As data was collected from NIS, all biases associated with retrospective studies are applicable to our study. Additionally, the hospitalizations selected for our study were based on the diagnosis of IBD rather than individual patients. Therefore, individuals admitted numerous times for the same chief compliant may have been included several times in the study population. Finally, NIS is an administrative database that uses a coding system to gather and store information; hence, there are possibilities for coding errors. However, despite these limitations, we believe that the large sample size, the specific study design, and a detailed analysis provide a better understanding of the topic in question. Through this study, we aimed to not only fill the gaps in literature that currently exist, but also promote intellectual conversation and promote future research on the topic.

In our study, the prevalence of obesity in IBD hospitalizations was noted to be $9.4 \%$ with a substantial female and White predominance. The mean age for IBD hospitalizations with obesity was found to be 47.9 years. Additionally, we noted higher prevalence of comorbidities such as diabetes mellitus, dyslipidemia, coronary artery disease, hypertension, congestive heart failure, chronic kidney disease, chronic obstructive pulmonary disease, and history of smoking in IBD hospitalizations with obesity compared to the non-obese cohort. Although IBD hospitalizations with obesity were noted to have higher mean THC and longer LOS, we did not find any statistical significance in the rates of mortality between the 2 subgroups. Compared to non-obese IBD hospitalizations, patient with concurrent obesity had higher rates of inpatient complications such as sepsis, AKF, ARF, and PE. In conclusion, obesity has a significant impact on IBD hospitalizations in terms of costs healthcare utilization and system-based complications.

\section{ADDITIONAL INFORMATION}

\section{Funding Source}

The authors received no financial support for the research, authorship, and/or publication of this article.

\section{Conflict of Interest}

No potential conflict of interest relevant to this article was reported. 


\section{Data Availability Statement}

We used the NIS database from 2016 to 2018, available online at http: //www.hcup- us.ahrq.gov. The NIS is a large publicly available, all-payer inpatient care database containing data on more than seven million hospital stays yearly in the US. Its large sample size is ideal for developing national and regional estimates and enables analyses of rare conditions, uncommon treatments, and special populations.

\section{Author Contribution}

Conceptualization: Dahiya DS. Data curation: Dahiya DS, Kichloo A. Formal analysis: Kichloo A. Investigation: Dahiya DS, Kichloo A, Wani F, Singh J, Shaka H. Methodology, project administration, resources, supervision: Dahiya DS, Kichloo A. Validation: Dahiya DS, Kichloo A, Wani F, Singh J, Shaka H. Visualization: Dahiya DS, Kichloo A. Writing - original draft: Dahiya DS, Kichloo A. Writing - review \& editing: all authors. Approval of final manuscript: all authors.

\section{ORCID}

Dahiya DS

https://orcid.org/0000-0002-8544-9039

Kichloo A https://orcid.org/0000-0003-4788-8572

Wani F https://orcid.org/0000-0002-4683-6845

Singh J https://orcid.org/0000-0001-7179-1020

Solanki D https://orcid.org/0000-0001-8655-225X

Shaka H https://orcid.org/0000-0002-9456-4581

\section{REFERENCES}

1. Fakhoury M, Negrulj R, Mooranian A, Al-Salami H. Inflammatory bowel disease: clinical aspects and treatments. J Inflamm Res 2014;7:113-120.

2. Lamb CA, Kennedy NA, Raine T, et al. British Society of Gastroenterology consensus guidelines on the management of inflammatory bowel disease in adults. Gut 2019;68(Suppl 3): s1-s106.

3. Lee SH, Kwon JE, Cho ML. Immunological pathogenesis of inflammatory bowel disease. Intest Res 2018;16:26-42.

4. GBD 2017 Inflammatory Bowel Disease Collaborators. The global, regional, and national burden of inflammatory bowel disease in 195 countries and territories, 1990-2017: a systematic analysis for the Global Burden of Disease Study 2017. Lancet Gastroenterol Hepatol 2020;5:17-30.

5. Centers for Disease Control and Prevention. Facts about IBD [Internet]. c2021 [cited 2021 Mar 13]. https://www.cdc.gov/ ibd/data-statistics.htm.
6. Pavelock N, Masood U, Minchenberg S, Heisig D. Effects of obesity on the course of inflammatory bowel disease. Proc (Bayl Univ Med Cent) 2019;32:14-17.

7. Szilagyi A. Relationship(s) between obesity and inflammatory bowel diseases: possible intertwined pathogenic mechanisms. Clin J Gastroenterol 2020;13:139-152.

8. Healthcare Cost and Utilization Project (HCUP). Introduction to the HCUP National Inpatient Sample (NIS), 2018 [Internet]. c2020 [cited 2021 Mar 13]. https://www.hcup-us.ahrq. gov/db/nation/nis/NIS_Introduction_2018.jsp.

9. Healthcare Cost and Utilization Project (HCUP). Nationwide Inpatient Sample (NIS) Redesign Final Report: HCUP Methods Series Report \# 2014-04 [Internet]. c2014 [cited 2021 Mar 13]. http://www.hcup-us.ahrq.gov/reports/methods/methods.jsp.

10. Healthcare Cost and Utilization Project (HCUP). Clinical Classifications Software Refined (CCSR) [Internet]. c2021 [cited 2021 Mar 13]. https://hcup-us.ahrq.gov/toolssoftware/ccsr/ ccs_refined.jsp.

11. Choi YJ, Choi EK, Han KD, et al. Increased risk of atrial fibrillation in patients with inflammatory bowel disease: a nationwide population-based study. World J Gastroenterol 2019;25: 2788-2798.

12. Knowles SR, Graff LA, Wilding H, Hewitt C, Keefer L, Mikocka-Walus A. Quality of life in inflammatory bowel disease: a systematic review and meta-analyses- part I. Inflamm Bowel Dis 2018;24:742-751.

13. Hammond RA, Levine R. The economic impact of obesity in the United States. Diabetes Metab Syndr Obes 2010;3:285-295.

14. Flegal KM, Carroll MD, Ogden CL, Curtin LR. Prevalence and trends in obesity among US adults, 1999-2008. JAMA 2010; 303:235-241.

15. Singh S, Dulai PS, Zarrinpar A, Ramamoorthy S, Sandborn WJ. Obesity in IBD: epidemiology, pathogenesis, disease course and treatment outcomes. Nat Rev Gastroenterol Hepatol 2017; 14:110-121.

16. Yanovski SZ, Yanovski JA. Obesity prevalence in the United States--up, down, or sideways? N Engl J Med 2011;364:987989.

17. Jura M, Kozak LP. Obesity and related consequences to ageing. Age (Dordr) 2016;38:23.

18. Hales CM, Carroll MD, Fryar CD, Ogden CL. Prevalence of obesity and severe obesity among adults: United States, 20172018. NCHS Data Brief 2020;(360):1-8.

19. Shah SC, Khalili H, Gower-Rousseau C, et al. Sex-based differences in incidence of inflammatory bowel diseases-pooled 
analysis of population-based studies from Western countries. Gastroenterology 2018;155:1079-1089.

20. Shi HY, Levy AN, Trivedi HD, Chan FKL, Ng SC, Ananthakrishnan AN. Ethnicity influences phenotype and outcomes in inflammatory bowel disease: a systematic review and meta-analysis of population-based studies. Clin Gastroenterol Hepatol 2018;16:190-197.

21. Abdelaal M, le Roux CW, Docherty NG. Morbidity and mortality associated with obesity. Ann Transl Med 2017;5:161.

22. Prospective Studies Collaboration, Whitlock G, Lewington S, et al. Body-mass index and cause-specific mortality in 900 000 adults: collaborative analyses of 57 prospective studies. Lancet 2009;373:1083-1096.

23. D'Souza MJ, Bautista RC, Wentzien DE. Data talks: obesity-related influences on US mortality rates. Res Health Sci 2018;3: 65-78.

24. Xu F, Wheaton AG, Liu Y, Lu H, Greenlund KJ. Hospitalizations for inflammatory bowel disease among medicare fee-for-service beneficiaries - United States, 1999-2017. MMWR Morb Mortal Wkly Rep 2019;68:1134-1138.

25. Johnson AM, Loftus EV Jr. Impact of obesity on the management of inflammatory bowel disease. Gastroenterol Hepatol 2020;16:350-359.

26. Seminerio JL, Koutroubakis IE, Ramos-Rivers C, et al. Impact of obesity on the management and clinical course of patients with inflammatory bowel disease. Inflamm Bowel Dis 2015; 21:2857-2863.

27. Xu F, Liu Y, Wheaton AG, Rabarison KM, Croft JB. Trends and factors associated with hospitalization costs for inflammatory bowel disease in the United States. Appl Health Econ Health Policy 2019;17:77-91.

28. Erlich J, Rubin DT. Predictors of IBD-related length of hospital stay and 30 day readmission in a tertiary center. Am. J Gastroenterol 2016;111:S277.

29. Healthcare Cost and Utilization Project (HCUP). NIS description of data elements [Internet]. c2021 [cited 2021 Apr 29]. https://www.hcup-us.ahrq.gov/db/vars/hosp_bedsize/nisnote.jsp.

30. Healthcare Cost and Utilization Project (HCUP). NIS description of data elements: Stratum used to post-stratify hospital [Internet]. c2021 [cited 2021 Apr 29]. https://www.hcup-us. ahrq.gov/db/vars/nis_stratum/nisnote.jsp.

31. Centers for Disease Control and Prevention. Data on obesity [Internet]. c2021 [cited 2021 Mar 13]. https://www.cdc.gov/ obesity/data/prevalence-maps.html.

32. Levine JA. Poverty and obesity in the U.S. Diabetes 2011;60: 2667-2668. 